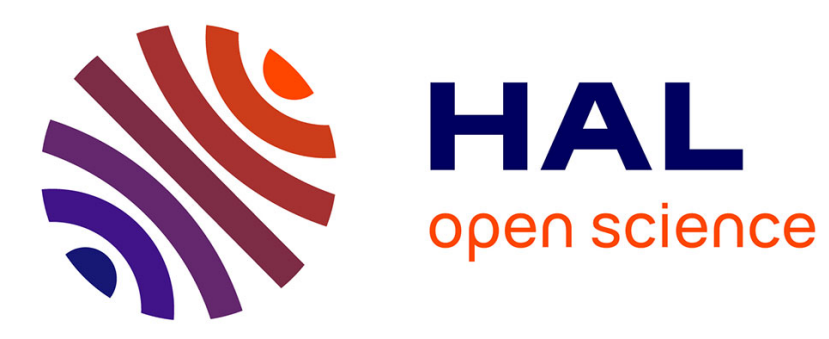

\title{
Physics of the sapphire whispering-gallery-mode solid-state MASER oscillator
}

Mohamad Mrad, Ahmad Tarhini, Pierre-Yves Bourgeois, Vincent Giordano

\section{To cite this version:}

Mohamad Mrad, Ahmad Tarhini, Pierre-Yves Bourgeois, Vincent Giordano. Physics of the sapphire whispering-gallery-mode solid-state MASER oscillator. European Physical Journal: Applied Physics, 2020, 91, pp.31001 (8). hal-02993778

\section{HAL Id: hal-02993778 \\ https://hal.science/hal-02993778}

Submitted on 7 Nov 2020

HAL is a multi-disciplinary open access archive for the deposit and dissemination of scientific research documents, whether they are published or not. The documents may come from teaching and research institutions in France or abroad, or from public or private research centers.
L'archive ouverte pluridisciplinaire HAL, est destinée au dépôt et à la diffusion de documents scientifiques de niveau recherche, publiés ou non, émanant des établissements d'enseignement et de recherche français ou étrangers, des laboratoires publics ou privés. 


\title{
PHYSIC OF THE SAPPHIRE WHISPERING-GALLERY-MODE SOLID-STATE MASER OSCILLATOR
}

\author{
M. Mrad ${ }^{1,2}$, A. Tarhini ${ }^{2}$, Pierre-Yves Bourgeois ${ }^{1}$, and Vincent Giordano ${ }^{1}$ \\ ${ }^{1}$ Laboratoire FEMTO-ST, 26 Chemin de l'Epitaphe, 25000 Besanon, France. \\ ${ }^{2}$ Department of Physics, Faculty of Sciences I \& V, Lebanese University, Hadat and Nabatieh, Lebanon.
}

\begin{abstract}
The Cryogenic Sapphire Oscillator (CSO) is currently the best available technology that can provide a relative frequency stability better than $10^{-15}$ for integration times between $1 \mathrm{~s}$ and $10,000 \mathrm{~s}$. But, the CSO remains a complex instrument requiring multiple loop controls to achieve the best frequency stability. The possibility to use of the sapphire resonator in a self-sustained MASER oscillator constitutes an elegant alternative to the CSO. Here, the sustaining amplification is achieved through the interaction between a high-Q factor whispering gallery mode and the paramagnetic $\mathrm{Fe}^{3+}$ ions, which are present in small concentration in the sapphire crystal. The $\mathrm{Fe}^{3+}$ ion exhibits three energy states enabling to realize a self-sustaining solid-state maser. Although, this principle has been already experimentally demonstrated few years ago, its development as a truly usable ultra-stable source has not yet been completed, mainly due to the lack of control of the complex physical phenomena involved. This paper completes the previous theoretical work based on the rate equations model. Here we derive the full quantum equations describing the evolution of the $\mathrm{Fe}^{3+}$ ions inside the sapphire lattice and submitted to a pump and a maser signal. The influence of the ions concentration and spin-spin relaxation time will be pointed out.
\end{abstract}

\section{INTRODUCTION}

The need for microwave sources exhibiting ultra-high spectral purity and high temporal stability is a key point highlighted by the performance limitations in major strategic applications such as radar, spatial communications, ultra-high accuracy navigation systems, and high bit rate optical telecommunications. The Cryogenic Sapphire Oscillator (CSO) is currently the only available technology able to provide a relative frequency stability better than $1 \times 10^{-15}$ for integration time between $1 \mathrm{~s}$ and $10^{4} \mathrm{~s}[1,2,3,4,5,6]$. The CSO is a classical transmission oscillator that uses as a frequency determining element a passive cryogenic high-Q sapphire resonator based on a cylindrical dielectric resonator made in a high purity sapphire monocrystal. To provide high Q-factor, the sapphire resonator is excited on a high order electromagnetic mode (Whispering Gallery mode or WG mode) and cooled down near the liquid helium temperature [7]. At $10 \mathrm{GHz}$, the Q-factor typically reaches $10^{9}$. Nevertheless, the CSO remains a complex instrument. For technical raisons, mainly to limit the required cooling power, the sustaining amplifier is placed outside the cryostat at room temperature. Two long microwave lines join the cryogenic resonator and the sustaining stage. Any variation in the phase or power of the signal injected in the resonator will produce an oscillator frequency variation limiting drastically the achievable frequency stability. To solve this issues, additional sophisticated electronical controls are mandatory to stabilize both the signal phase and power.

In 1959, Bogle and Symmons [8] discussed in detail the study of the resonance of $\mathrm{Fe}^{3+}$ in alumina and presented accurate measurements of transition frequencies. We reported in $[9,10]$ the possibility to use the sapphire resonator in another way. Here, the sustaining amplification is achieved through the interaction between a high-Q factor whispering gallery mode and the paramagnetic $\mathrm{Fe}^{3+}$ ions, which are present in small concentration in the sapphire crystal. The $\mathrm{Fe}^{3+}$ ion exhibits three energy states enabling to realize a self-sustaining solid-state maser. The population inversion is obtained by submitting the $\mathrm{Fe}^{3+}$ ions to a $31 \mathrm{GHz}$ signal. The maser oscillation takes place at $12.04 \mathrm{GHz}$, i.e. the ground state electron spin resonance (ESR) frequency of $\mathrm{Fe}^{3+}$. As the sapphire resonator whispering gallery mode exhibits at low temperature extremely low losses, the $\mathrm{Fe}^{3+}$ ions concentration required to get enough gain is very low : typically, below 1 ppm (part-per-million).

Since the experimental demonstration of this new type of solid-state maser [11, 12, 13, 14], few theoretical works have been published to explain its main features $[15,16,17]$. Particularly, the effectiveness of the maser process, i.e. the output 


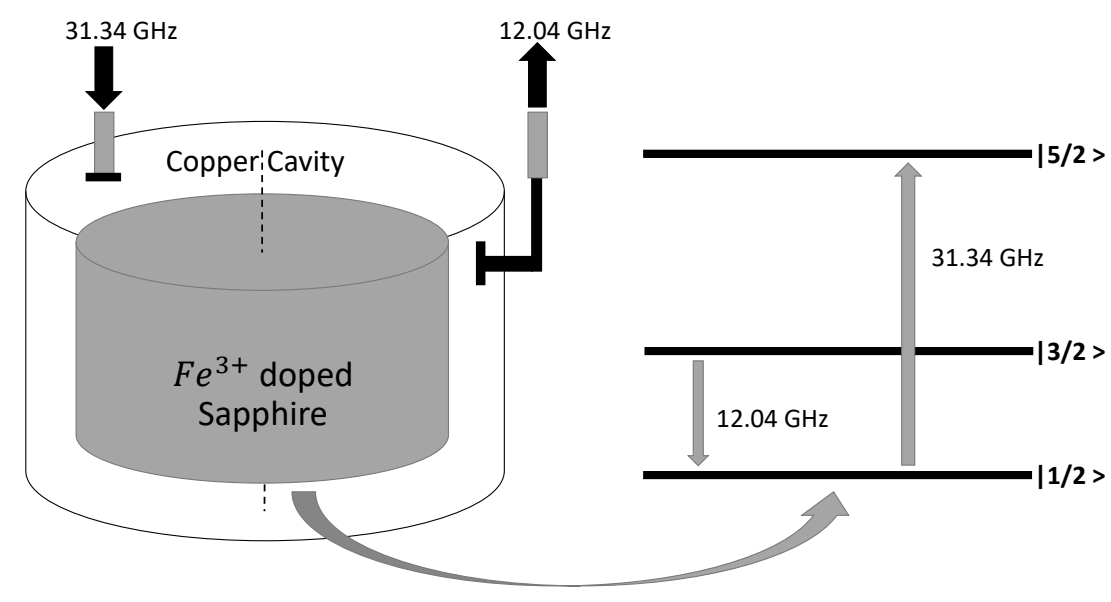

FIGURE 1. Bloembergen's three-level sapphire maser.

power as a function of the pumping power and the resonator coupling has been derived by using the classical rate equations model [18] as stated by Siegman in the early 60s.

In this paper, we derive the exact equations describing the sapphire maser operation using a quantum mechanics approach considering the coherences that are created between the different quantum states of the paramagnetic ion. These coherences were assumed negligible in the previous approach based on the rate equations. The effect on the maser operation of these coherences increases as a function of the spin-spin relaxation time and of dopant concentration. Conversely, it decreases as a function of the spin-lattice relaxation time.

\section{THE WG MODE MASER OSCILLATOR}

$\mathrm{Fe}^{3+}$ ions are present in high quality sapphire Hemex crystals in quantities of few parts per million. The $\mathrm{Fe}^{3+}$ ion in the sapphire lattice presents 3 energy levels at zero-magnetic-field $[19,20]$ corresponding to the classical Bloembergen 3 levels scheme [21] represented in Figure 1. To create the inversion population between the first two levels, Fe ${ }^{3+}$ ions are pumped to the third level by applying a microwave signal $31.34 \mathrm{GHz}$. Then relaxation occurs between $E_{3}$ (i.e. state $\mid 5 / 2>$ ) and $E_{2}$ (i.e. state $\mid 3 / 2>$ ) causing population inversion between states $\mid 1 / 2>$ and $\mid 3 / 2>$. The system is able to amplify signal at the $\mathrm{Fe}^{3+}$ EPR frequency, i.e. $12.04 \mathrm{GHz}$. If the amplifier gain is sufficient to compensate for the resonator loss, the system becomes self sustained and a stable $12.04 \mathrm{GHz}$ can be extracted from the cryogenic oscillator.

The Whispering Gallery Mode Maser prototype consists in a cylindrical sapphire resonator, $50 \mathrm{~mm}$ diameter and $30 \mathrm{~mm}$ thick. It is placed in the center of a cylindrical cavity and this assembly is cooled down the liquid helium temperature. The resonator $W \mathrm{GH}_{17,0,0}$ mode frequency coincides with the $\mathrm{Fe}^{3+} \mathrm{ESR}$, i.e. $12.04 \mathrm{GHz}$. Around the pump signal frequency, i.e. $31.3 \mathrm{GHz}$, the resonator mode spectrum is dense and several high-Q factor modes liying in the ESR bandwidth can be used to pump the ions and get oscillation [22,23]. Depending on the coupling factors, the chosen pump mode and the active ions concentration, the maser signal power detected outside the cryostat can be as high as $-30 \mathrm{dBm}$.

\section{FE $^{3+}$ IONS PARAMETERS IN THE SAPPHIRE CRYSTAL}

The maser operation depends on the concentration $\mathrm{N}$ and relaxation times of the $\mathrm{Fe}^{3+}$ paramagnetic ions. In our experiments, the sapphire resonator are machined in high quality crystals HEMEX type [24] containing very small amount of defect and paramagnetic impurities. This situation greatly differs from those of previous works undertaken in the 60's for which crystal quality was poor compared to the current technology. 


\section{1. $\mathbf{N}$ effective ions concentration :}

Quantitative ionic impurities concentration measurements have been conducted on HEMEX crystals with different methods [25]. All these measurements agree within the resolution measurements and give a total iron ion concentration at a level of $2 \mathrm{ppm}$. The given concentration corresponds to the total amount of iron ions including both $\mathrm{Fe}^{2+}$ and $\mathrm{Fe}^{3+}$, among which only the latter is paramagnetic and gives rise to the maser effect. The effective concentration of $\mathrm{Fe}^{3+}$ ions has been evaluated in several resonators by measuring the real part of the magnetic susceptibility [26]. It appears the number of ions that effectively contribute, is very small, i.e. $\mathrm{N}$ is typically in the range $10-20$ parts-per-billion (10-20 ppb). This low value is compatible with the maser signal power experimentally observed [24]. It indicates that in typical HEMEX crystals, $\mathrm{Fe}^{2+}$ dominates. This was confirmed in $2000[27]:$ a conversion $F e^{2+}$ to $\mathrm{Fe}^{3+}$ was achieved by thermally annealing a HEMEX resonator [28] in air resulting in a large increase of the effective ion concentration and consequently of the maser signal power [26]. This led to assume that the $\mathrm{Fe}^{3+}$ concentration that can be found in high quality sapphire crystal should be in the range :

$$
10 p p b<N<300 p p b
$$

\section{2. $\tau_{1}$ longitudinal or spin-lattice relaxation time :}

$\tau_{1}$ describing the time required for the populations to recover their thermal equilibrium after any perturbation. The spinlattice relaxation time for $\mathrm{Fe}^{3+}$ in sapphire has been measured by several groups [8, 24, 29] and at the liquid helium temperature is in the range :

$$
5 m s<\tau_{1}<20 m s
$$

\section{3. $\tau_{2}$ transversal or spin-spin relaxation time :}

$\tau_{2}$ is the rate at which, the transverse component of the magnetization, exponentially decays towards zero. $\tau_{2}$ is phenomenologically introduced in the rate equation model as related to the ESR linewidth by $: \Delta v=1 / \pi \tau_{2}$. The transversal relaxation time is ultimately limited by the interaction between neighboring spins : spin-to-spin coupling. According to the Van Vleck's theory [30], the ESR linewidth resulting from the dipole coupling should be proportional to the ions concentration $\mathrm{N}$ :

$$
\Delta v \approx 6 g^{2} \beta^{2} \mu_{0} N \sqrt{S(S+1)} / h
$$

where $\mathrm{g}$ is Landé $\mathrm{g}$-factor, $\beta$ the Bohr magneton, $\mu_{0}$ permeability constant and the spin $\mathrm{S}=5 / 2$. Applied to the Fe $\mathrm{F}^{3+}$ ions, the equation (3) was only approximately verified for relatively high concentrations [8, 20]. For high quality sapphire crystals observed $\Delta v$ are always several tens of $\mathrm{MHz}$ wide, and thus can not be attributed to the spin-to-spin coupling nor to the crystal defects, but to the hyperfine interaction between $\mathrm{Fe}^{3+}$ ion and $\mathrm{Al}$ nuclei that surround it.

If the crystal is perfectly homogeneous and presents homogeneous distribution of ions, then the lineshape is determined by the relaxation times and therefore lorentzian lineshapes are a common result. The EPR spectrum is the sum of a large number of lines each having the same Larmor frequency and linewidth. In general, the ions are not all in the same environment, the lineshape is determined by unresolved couplings because the EPR spectrum is the sum of a large number of narrower individual homogeneously broadened lines that are each shifted in frequency with respect to each other [20, 24, 26].

$$
1 n s<\tau_{2}<20 n s
$$

\section{MASTER EQUATION FOR FE FE $^{3+}$}

$\rho(t)$ is the density matrix operator. The diagonal elements $\rho_{i i}$ or $n_{i}$ are the energy level populations, which at thermal equilibrium reduces to $N_{i}$ with :

$$
\frac{N_{j}}{N_{i}}=\exp \left(-\frac{h v_{i j}}{k_{B} T}\right)=e^{-\Delta_{i j}} \quad \text { and } \quad \sum_{i=1}^{3} N_{i}=1
$$

where $v_{i j}$ is the Bohr frequency. We will deal with the varying population differences $: n_{i j}=\rho_{i i}-\rho_{j j}$. 
The off-diagonal elements $\rho_{i j}=\langle i|\rho| j\rangle,(i \neq j)$ represent the coherence between levels $\mid i>$ and $|j\rangle$. We assume solutions of the form :

$$
\rho_{12}=\delta_{12} e^{i \omega_{21} t} ; \rho_{13}=\delta_{13} e^{i \omega_{31} t} ; \rho_{23}=\delta_{23} e^{i \omega_{32} t}
$$

where $\delta_{i j}$ are complex slow time-varying functions. The master Liouville-Bloch equations of the system are [31] :

$$
\begin{aligned}
\frac{d n_{1}}{d t} & =-\frac{n_{12}-N_{12}}{2 \tau_{1}^{(12)}}-\frac{n_{13}-N_{13}}{2 \tau_{1}^{(13)}}-\Omega_{R p} \operatorname{Im}\left[\delta_{13}\right]-\Omega_{R s} \operatorname{Im}\left[\delta_{12}\right] \\
\frac{d n_{2}}{d t} & =\frac{n_{12}-N_{12}}{2 \tau_{1}^{(12)}}-\frac{n_{23}-N_{23}}{2 \tau_{1}^{(23)}}+\Omega_{R s} \operatorname{Im}\left[\delta_{12}\right] \\
\frac{d n_{3}}{d t} & =\frac{n_{13}-N_{13}}{2 \tau_{1}^{(13)}}+\frac{n_{23}-N_{23}}{2 \tau_{1}^{(23)}}+\Omega_{R p} \operatorname{Im}\left[\delta_{13}\right] \\
\frac{d \delta_{12}}{d t} & =-\frac{\delta_{12}}{\tau_{2}^{(12)}}+i \frac{\Omega_{R s}}{2} n_{12}-i \frac{\Omega_{R p}}{2} \delta_{23}^{*} \\
\frac{d \delta_{13}}{d t} & =-\frac{\delta_{13}}{\tau_{2}^{(13)}}-i \frac{\Omega_{R s}}{2} \delta_{23}+i \frac{\Omega_{R p}}{2} n_{13} \\
\frac{d \delta_{23}}{d t} & =-\frac{\delta_{23}}{\tau_{2}^{(23)}}-i \frac{\Omega_{R s}}{2} \delta_{13}+i \frac{\Omega_{R p}}{2} \delta_{12}^{*}
\end{aligned}
$$

where, $\delta_{i j}^{*}=$ Conjugate $\left[\delta_{i j}\right]$. The factors $\tau_{1}^{(i j)}$ and $\tau_{2}^{(i j)}$ are respectively the spin-lattice relaxation time and the spin-spin relaxation time of the pair of levels $(i, j)$.

Pace [32] introduced measures showing that relaxation times are of the same order of magnitude. We therefore assume that $\tau_{1}^{(12)}=\tau_{1}^{(23)}=\tau_{1}^{(13)}=\tau_{1} \forall \mathrm{i}$ and $\mathrm{j}$.

Similarly for the spin-spin relaxation time, we therefore assume that, $\tau_{2}^{(12)}=\tau_{2}^{(23)}=\tau_{2}^{(13)}=\tau_{2} \forall \mathrm{i}$ and $\mathrm{j}$.

The resolution of the previous system of equations is complex, Therefore we'll focus only the steady state of the system :

$$
d n_{i} / d t=0 \text { and } d \delta_{i j} / d t=0
$$

In addition, the equations are not independent of each other. A seventh equation is necessary. It corresponds to the sum of population differences such as :

$$
n_{12}+n_{23}-n_{13}=0
$$

The relationship [24, 31] between $\Omega_{R p}^{2}$ and $W_{P}$ is $\Omega_{R p}^{2}=2 W_{P} / \tau_{2}$, where $W_{P}$ is the absorption probability (same relationship between $\Omega_{R s}^{2}$ and $W_{S}$ with that $W_{S}$ is the emission probability).

The stationary solutions of the general model are the following :

$$
\begin{gathered}
n_{12_{a c}}=\frac{a\left(3+2 \tau_{1} W_{P}\right) N_{12}-2 b_{S} \tau_{1} W_{P} N_{23}}{6+c+d+4 \tau_{1}\left(2 \tau_{1}-\tau_{2}\right) W_{P} W_{S}} \\
n_{13_{a c}}=\frac{a\left(3+2 \tau_{1} W_{S}\right) N_{13}+2 b_{P} \tau_{1} W_{S} N_{23}}{6+c+d+4 \tau_{1}\left(2 \tau_{1}-\tau_{2}\right) W_{P} W_{S}} \\
\text { where: }\left\{\begin{array}{r}
a=2+\tau_{2}\left(W_{S}+W_{P}\right) \\
b_{S}=2+\tau_{2}\left(W_{P}-2 W_{S}\right) \\
b_{P}=2+\tau_{2}\left(W_{S}-2 W_{P}\right) \\
c=\left(8 \tau_{1}+3 \tau_{2}\right)\left(W_{P}+W_{S}\right) \\
d=4 \tau_{1} \tau_{2}\left(W_{P}^{2}+W_{S}^{2}\right)
\end{array}\right.
\end{gathered}
$$

To derive the rate equations model (Without coherence) from the general model, we eliminate the terms of coherence, assuming that the coherence time $\tau_{2}$ tends to zero. The stationary solutions of the rate equations model are :

$$
n_{12_{s c}}=\frac{2 \tau_{1}\left(N_{12}-N_{23}\right) W_{P}+3 N_{12}}{4 \tau_{1}\left(1+\tau_{1} W_{S}\right) W_{P}+4 \tau_{1} W_{S}+3}
$$




$$
n_{13_{s c}}=\frac{2 \tau_{1}\left(N_{13}+N_{23}\right) W_{S}+3 N_{13}}{4 \tau_{1}\left(1+\tau_{1} W_{S}\right) W_{P}+4 \tau_{1} W_{S}+3}
$$

\section{THE POWERS}

\subsection{Evolution of the emitted power as a function of pump power}

In this paragraph we will defined the relation at the resonator input between the pump power $\left(P_{p}\right)$ and the maser power $\left(P_{S}\right)$. The power that can be emitted by a maser system is define as [18] :

$$
P_{s}=h v_{12} W_{S} n_{21} V_{e f f_{12}}
$$

$V_{e f f_{12}}$ is the effective volume of the whispering gallery mode involved in the emmission process [15]. The emission probability is represented as follow [18] :

$$
W_{S}=\frac{1}{4} \gamma^{2} g_{\text {signal }}(v) Q_{0_{S}} H_{\text {acsignal }}^{2} \sigma_{12}^{2}
$$

From the definition of the Poynting vector, the power in the resonator is directly proportional to the magnetic field. It leads to :

$$
H_{\text {acsignal }}^{2}=\frac{2 P_{s}}{Z_{c} S_{e f f_{12}}}
$$

Therefore :

$$
W_{S}=\gamma^{2} \tau_{2}^{(12)} Q_{0_{S}} \frac{\sigma_{12}^{2}}{Z_{c} S_{e f f_{12}}} P_{s}=w_{S_{0}} P_{s}
$$

This representation can also be applied to the absorption probability :

$$
W_{P}=\gamma^{2} \tau_{2}^{(13)} Q_{0_{P}} \frac{\sigma_{13}^{2}}{Z_{c} S_{e f f_{13}}} P_{p}=w_{P_{0}} P_{p}
$$

$\gamma$ is the gyromagnetic factor. $S_{\text {eff }}$ and $S_{\text {eff }}$ are respectively the effective surface of the whispering gallery mode in the meridian resonator plan for the maser signal and the maser pump. $Z_{c}$ is the characteristic resonator impedance, $\sigma_{i j}^{2}$ is the transition parameter between the two levels $i$ and $j$, which their value can be obtained through the calculation of the spin Hamiltonian. $Q_{0_{S}}$ and $Q_{0_{P}}$ are the quality factors of the whispering gallery modes for the maser signal (the ions from the second to the first level) and the maser pump (the ions from the first to the third level) respectively [15].

To remain in the validity field, we neglect the second order term and suppose that $\tau_{2}<<\tau_{1}$ and write $C=h v_{12} N V_{e f f_{12}} w_{S_{0}}$. From equations $\left(9 \mathrm{a}, 11,14\right.$ and 15) we can now determined the maser power $\left(P_{s}\right)$ as a function of the pump power $\left(P_{p}\right)$ :

$$
\begin{gathered}
P_{S}=\frac{1}{w_{S_{0}}} \frac{\left(-1+\frac{1}{2} C\left(N_{23}-N_{12}\right)\right) P_{p}-\frac{3}{4 \tau_{1}{ }^{w} P_{0}}\left(1+C N_{12}\right)}{\frac{1}{{ }^{{ } P_{0}}}+\tau_{1}(1+x) P_{p}} \\
x=\left\{\begin{array}{cl}
0 & \text { Without Coherence } \\
\frac{C \tau_{2}}{4 \tau_{1}}\left(N_{13}+N_{23}\right) & \text { With Coherence }
\end{array}\right.
\end{gathered}
$$

For the production of a maser, we need to calculate the threshold pump power and maximum power of the maser in the resonator. 


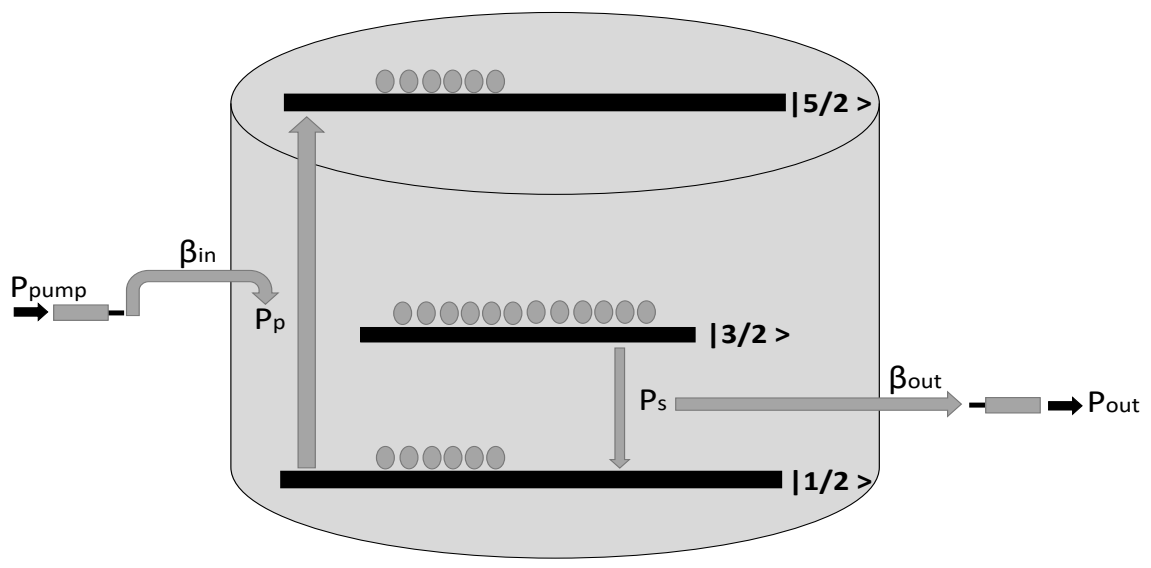

FIGURE 2. Schematic of stimulated emission at $12.04 \mathrm{GHz}$.

\section{2. threshold Pump power}

The pump power threshold $P_{p_{T h}}$ is when the population inversion occurs, that is to say, when $n_{12}=0$. The rate absorption threshold $\left(W_{P_{T h}}\right)$ can be written,

$$
W_{P_{T h}}=\frac{3}{2 \tau_{1}} \frac{N_{12}}{N_{23}-N_{12}}
$$

Then, substituting the expressions of $W_{P_{T h}}$ (equation (17)) and $n_{13_{T h}}$ (equation (9b) or (10b)) in the expression for the power pump :

$$
P_{p_{T h}}=\frac{3}{2 \tau_{1}} h v_{13} N_{12} N V_{e f f_{13}}
$$

\subsection{Maximum output power}

If the power applied to pump ions continues to increase, the system will eventually become saturated. In another form, the power $P_{s}$ in a resonator will be maximum when the pump has a power strong enough. Using the equation (16), the maximum power of the signal maser reads :

$$
P_{s_{\max }}=\lim _{P_{p} \rightarrow \infty} P_{s}=\frac{C}{2 \tau_{1} w_{S_{0}}} \frac{N_{23}-N_{12}}{1+x}
$$

\subsection{Outside the resonator}

The injected power $\left(P_{\text {pump }}\right)$ is recycled by a high-order whispering gallery mode with a coupling coefficient $\beta_{\text {pump }}$. For [15], the transmitted pump power can be expressed as [18] :

$$
P_{p}=\left(1-\left(\frac{1-\beta_{\text {pump }}}{1+\beta_{\text {pump }}}\right)^{2}\right) P_{\text {pump }}
$$

The output power due to stimulated emission of the ions will be recycled within the $\mathrm{WG}_{17,0,0}$ mode centered on the $\mathrm{Fe}^{3+}$ EPR due to the high Q-factor, a schematic is shown in figure 2. The output power $\left(\mathrm{P}_{\text {out }}\right)$ is a function of the different losses and gain of the three-level spin system and also of the whispering gallery mode characteristics. For a maser oscillator this output power can be written as a function of the output coupling $\beta_{\text {out }}$ :

$$
P_{\text {out }}=\frac{\beta_{\text {out }}}{\beta_{\text {out }}+1} P_{\text {max }}
$$

with $\beta_{\text {out }}$ the coupling coefficient at the output of the maser. 


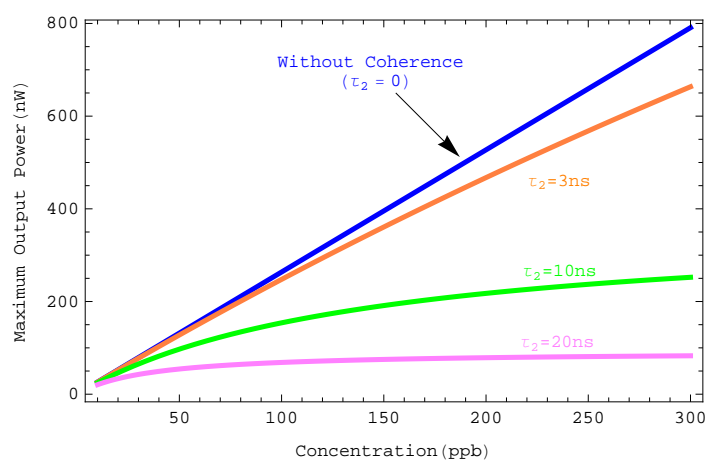

FIGURE 3. Evolution of the maximum output power $(n W)$ versus concentration for three values of $\tau_{2}: 3 n s, 10 n s$ and 20 ns. $\tau_{1}$ was assumed to be $10 \mathrm{~ms}$.

\section{RESULTS AND DISCUSSION}

To determine an order of these powers, we will consider the parameters at $4.2 \mathrm{~K}$ of a resonator. The Bohr frequencies are $v_{12}=12.04 \mathrm{GHz}$ and $v_{13}=31.32 \mathrm{GHz}$. At thermal equilibrium we have $: N_{12}=0.050, N_{23}=0.067$ and $N_{13}=0.117$. The values of the transition parameters $\sigma_{i j}^{2}$ for $\mathrm{Fe}^{3+}$ paramagnetic ion in the sapphire are $\sigma_{12}^{2}=2$ and $\sigma_{13}^{2}=0.0026$ [8]. The total volume of cristal is equal $V=58.9 \mathrm{~cm}^{3}$. According [33], the effectif volumes of the WGM are $V_{\text {eff } f_{12}} \approx \frac{2 V}{7}$ and $V_{\text {eff }} \approx \frac{2 V}{17}$. The quality factors are equal $Q_{0_{S}}=6 \times 10^{8}, Q_{0_{P}}=10^{8}$, the value of the characteristic resonator impedance is $Z_{c}=119 \Omega$. To understand the influence of the ions concentration and coherence time on our models, we plot the maximum emitted power as a function of concentration for three values of $\tau_{2}$. The results are given in the Figure 3 .

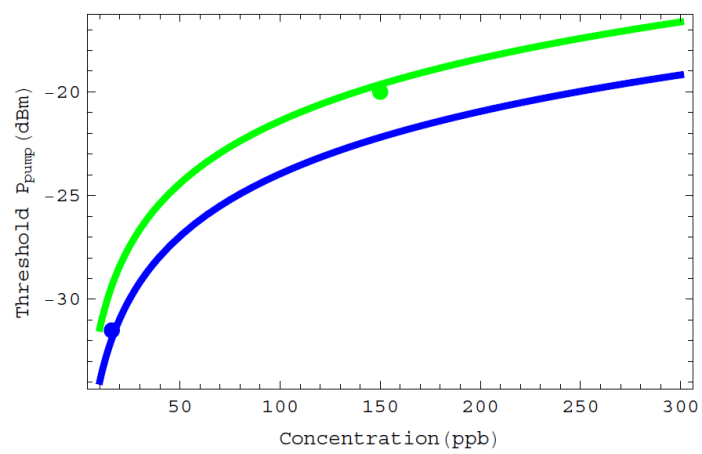

FIGURE 4. Evolution of the Threshold pump power versus concentration.

The coherence time $\tau_{2}$ is limited by the interaction between neighboring spins (spin-spin coupling). When the return to the equilibrium of the transverse magnetization component is slow, i.e. long coherence time, the power that can be extracted from the maser is lower than in absence of spin-to-spin coupling.

In order to verify the preceding expectations, we have tested three different resonators with different values of the parameters $N, \tau_{1}, \tau_{2}$. The resonator is loaded into a copper cavity and then cooled to the liquid helium temperature in a cryostat. The maser output power is measured with a microwave power spectrum analyzer while the injected power is measured with a power meter. The characteristics of the three resonators are, Pinocchio (16ppb, $18 \mathrm{~ms}, 2 \mathrm{ns)} \mathrm{[15,} \mathrm{24],} \mathrm{Jeanne} \mathrm{(11}$ ppb, $18 \mathrm{~ms}, 2 \mathrm{~ns}$ ) [15, 24], and a particular resonator dubbed Geppetto (150 ppb, $7 \mathrm{~ms}, 5.9 \mathrm{~ns})$ [26]. In figure 4, we plot the threshold Pump power for the first and the third resonator from equation 20.

In figure 5 we plot the theoretical results (with and without coherence) and the experimental data from equation 21.

Experimentally, the cryogenic resonator is connected to room temperature via a microwave K-cable with $5 \mathrm{~dB}$ loss. The resonator is loaded into a copper cavity and then cooled to the liquid helium temperature in a cryostat. Two microwave cables enable us to inject the pump power in the cryogenic sapphire resonator $(5 \mathrm{~dB}$ loss $)$ and to extract the maser output power (the data of the first and second resonator are $2 \mathrm{~dB}$ loss). Therefore, these losses have been deducted from the theoretical curve to consider the microwave cable losses. 

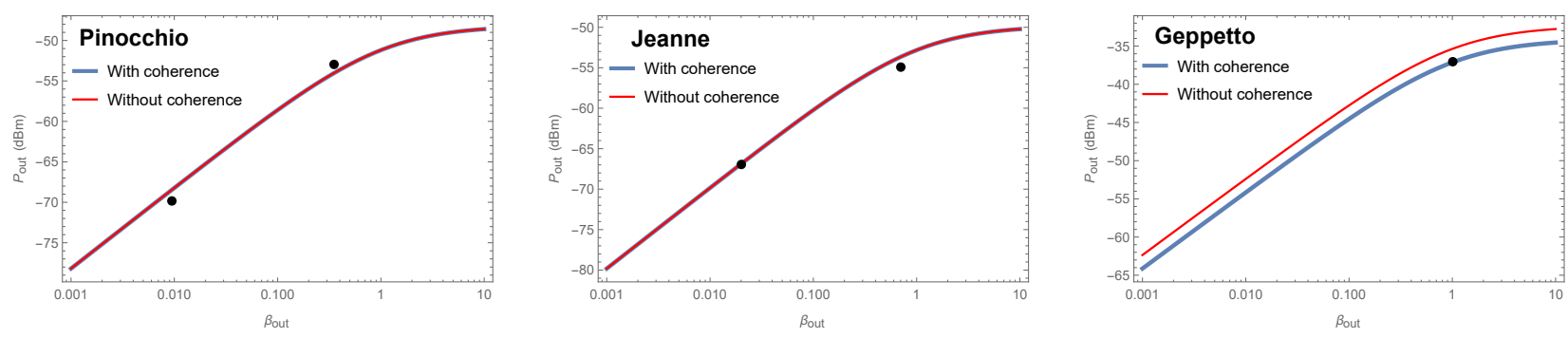

FIGURE 5. Maser output power as a function of the output coupling $\beta_{\text {out }}$ for three resonators.

\section{APPLICATION TO OTHER IONS}

In the case of $\mathrm{Fe}^{3+}$, the effect of coherence is limited. Other paramagnetic ions, as $\mathrm{Mo}^{3+}$ and $\mathrm{Cr}^{3+}$, can validate the difference between the two models, using a static magnetic field.

Cavity masers using ruby were developed at the Jet Propulsion Laboratory (JPL) at 960 megahertz (MHz) and at 2388 MHz. The 2388-MHz maser was used to receive and amplify microwave radar echoes from the planet Venus [34]. The total number of spins in pink ruby crystal with slightly more than 0.05 percent $\mathrm{Cr}^{3+}$ is about $2.5 \times 10^{25}$ per cubic meter. A magnetic field strength of 2500 gauss $(\mathrm{G})$ is applied to the ruby in a direction that is perpendicular to the c-axis. The four ground-state microwave spin levels that occur are called paramagnetic levels or Zeeman levels. The population inversion (maser signal) between levels 2 and 1, resulting from pumping between levels 1 and 4 .

By repeating the previous calculation, but respecting the new conditions, we find the maximum power of the signal maser :

$$
\begin{gathered}
P_{s_{\max }}=\lim _{P_{p} \rightarrow \infty} P_{s}=\frac{4 C}{9 \tau_{1} w_{S_{0}}} \frac{N_{24}-N_{12}}{1+x} \\
x=\left\{\begin{array}{cl}
\text { for } P_{s c} \text { (Without Coherence) } \\
\frac{2 C \tau_{2}}{9 \tau_{1}}\left(N_{14}+N_{24}\right) & \text { for } P_{a c} \text { (With Coherence) }
\end{array}\right.
\end{gathered}
$$

In this case, the Bohr frequencies are $v_{12}=2.398 \mathrm{GHz}, v_{13}=12.88 \mathrm{GHz}$ and $v_{14}=24.44 \mathrm{GHz}$. At thermal equilibrium ( $\mathrm{T}=$ $4.2 \mathrm{~K}$ ) we have : $N_{12}=0.0075, N_{13}=0.038$ and $N_{14}=0.068$. The values of the transition parameters $\sigma_{i j}^{2}$ for $C r^{3+}$ paramagnetic ion in the Ruby are $\sigma_{12}^{2}=1.59, \sigma_{13}^{2}=0.59$ and $\sigma_{14}^{2}=0.076$.

For different ions in crystals, we present in Table 1 the values of the spin-lattice and spin-spin relaxation times, the concentrations, the parameter $x$, the calculated and experimental powers. In that case, the coherences will have a large effect on the maser functioning as showed by the parameter $x$.

TABLE 1. Application of the model on other ions.

\begin{tabular}{|c|c|c|c|c|c|c|c|}
\hline Ion in crystal & $\tau_{2}(\mathrm{~ns})$ & $\tau_{1}(\mathrm{~ms})$ & $\mathrm{N}(\mathrm{ppb})$ & $x$ & $P_{s c}(\mathrm{dBm})$ & $P_{a c}(\mathrm{dBm})$ & $P_{\exp }(\mathrm{dBm})$ \\
\hline $\mathrm{Fe}^{3+}$ in Pinocchio & 2 & 18 & 16 & 0.0043 & -52.03 & -52.05 & $-53[15]$ \\
\hline $\mathrm{Fe}^{3+}$ in Geppetto & 5.9 & 7 & 150 & 0.5 & -35.36 & -37.13 & $-37[26]$ \\
\hline $\mathrm{Cr}^{3+}$ in Ruby & $60[35]$ & $157[36]$ & 500000 & 605 & -10.43 & -38.26 & $-40[37]$ \\
\hline $\mathrm{Mo}^{3+}$ in Sapphire & $>21[38]$ & $<0.1[39]$ & 300 & $>500$ & - & - & - \\
\hline
\end{tabular}

\section{CONCLUSION}

In this paper, we apply a full quantum model to the paramagnetic impurities that can used to design a maser source based on a high-quality sapphire monocrystal. We show that the maser power decreases as the coherence time $\tau_{2}$ increases. The rate equation model predicts that the maser power linearly with paramagnetic dopant concentration N. Our model including coherence predicts a more realistic behavior for the maser power that converges to a limit independent of $N$. 


\section{RÉFÉRENCES}

[1] C. Fluhr, S. Grop, B. Dubois, Y. Kersal, E. Rubiola and V. Giordano, IEEE Trans., 2016, Ultrasonics, Ferroelectrics and Frequency Control, 63, 6, 915-921

[2] V. Giordano, S. Grop, C. Fluhr, B. Dubois, Y. Kersal, E. Rubiola., 2016, Journal of Physics : Conference Series, 723 , $12-30$

[3] C. Fluhr, B. Dubois, S. Grop, J. Paris, G. Le Tet, V. Giordano, Dec., 2016, Cryogenics, 80, 164-175

[4] C. E. Calosso, F. Vernotte, V. Giordano, C. Fluhr, B. Dubois, E. Rubiola, 2019, IEEE Tran. on Ultrasonics, Ferroelectrics and Frequency Control, 66, 3, 616-623.

[5] C. R. Locke, E. N. Ivanov, J. G. Hartnett, P. L. Stanwix, and M. E. Tobar, 2008, Review of Scientific Instruments 79, 051301.

[6] [6] J.G. Hartnett and N.R. Nand, IEEE Trans. On Microwave Theory and Techniques, 2010, 58, 12, 3580-3586.

[7] Chang S., Mann A. G., Luiten A. N., and Blair D. G., 1997, PRL, 79, 2141

[8] Bogle G. S. and Symmons H. F., 1959, Proc. Phys. Soc., 73, 531

[9] Bourgeois P.Y., Bazin N., Y. Kersalé, V. Giordano, M.E. Tobar, and M. Oxborrow, 2005, Proc. 19th EFTF Besançon, 134-137

[10] P.Y. Bourgeois, N. Bazin, Y. Kersalé, V. Giordano, M.E. Tobar, and M. Oxborrow, 2005, APL, 87(22), 224104-1-3

[11] Benmessai K., Bourgeois P.Y., Kersalé Y., Bazin N., Tobar M.E., Hartnett J G, Oxborrow M, and Giordano V., 2007, Electron. Lett., 43, 1436

[12] Benmessai K., Creedon D. L., Tobar M. E., Bourgeois P. Y., Kersale Y., and Giordano V., 2008, PRL, 100, 233901

[13] M. Mrad, Y. Kersale, P.-Y. Bourgeois V. Giordano, S. Grop K. Benmessai and M.-E. Tobar, April 2012, IEEE-EFTF conference, Gothenburg, Sweden.

[14] Alexandre Didier, Jacques Millo, Clment Lacrote, Morvan Ouisse, Jrme Delporte, Vincent Giordano, Enrico Rubiola and Yann Kersal, 2016, Journal of Physics : Conference Series. 723, 012029,

[15] Benmessai K., Bourgeois P.Y., Tobar M.E., Bazin N., Kersalé Y., and Giordano V., 2010, Meas. Sci. Technol., 21, 025902

[16] M. Mrad, P.Y. Bourgeois, M.E. Tobar, Y. Kersal and V. Giordano, 2012, Eur. Phys. J. Appl. Phys. 57 : 21005

[17] Farr W. G., Goryachev M., Creedon D. L. and Tobar M. E., 2014, PRB, 90, 054409

[18] Siegman A. E., 1964, Microwave Solid State Masers, New York : McGraw-Hill

[19] Kornienko L. S. and Prokhorov A. M., 1961, JETP, 13

[20] Symmons H. F. and Bogle G. S., 1962, Proc. Phys. Soc., 79, 468

[21] Bloembergen N.,1965, Phys. Rev., 104, 324

[22] Tobar, M. E., and Mann, A. G., 1991. IEEE Tran. Microw. Theor. Tech., 39, 2077

[23] Wolf P., Tobar M. E., Bize S., Clairon A., Luiten A. N. and Santarelli G., 2004, Gen. Rel. Grav., 36, 2351

[24] Benmessai K., 2008, PhD thesis, Franche-comté University

[25] Khattak C. P. and al., 2001, Journal of Crystal Growth, 225, 572-579

[26] Creedon D. L., Benmessai K., Tobar M. E., Hartnett J. G., Bourgeois P.Y., Kersalé Y., Le Floch J. M., and Giordano V., 2010, IEEE Trans. Ultrason., Ferroelect., Frequency Control. 57, 3, 641646

[27] Benabid F., Notcutt M., Loriette V., Ju L., and Blair D. G., 2000, J. Phys. D, 33, 6, 589-594

[28] Emmett J. L. and Douthit T. R., 1993, Gems Gemol., 27, 4, 250-272

[29] Kornienko L. S. and Prokhorov A. M., 1959, Sov.Phys., 36, 649

[30] Van Vleck J. H., 1948, Phys. Rev., 74, 9, 1168-1183

[31] Godone A., 2002, Coherent Population Trapping Maser, Instituto Elettrotecnico Nazionale G. Ferraris

[32] Pace J.H., Sampson D.F., and Thorp J.S., 1961, Proc. Phys. Soc., 76, 697

[33] Cros D., 1990, PhD thesis, Limoges university

[34] W. R. Corliss, 1976, A History of the Deep Space Network, NASA CR-151915.

[35] S. CLEMENT, 1967, Contribution ltude des rubis : Expriences de R.P.E. Revue de Physique Applique (Paris), $2: 97100$.

[36] J.C. Jezequel, 1969 Mesure des temps de relaxation spin-rseau du rubis avec des structures maser en transmission. Revue de Physique Applique (Paris), 4 :341343. 
[37] Macgregor S. Reid, 2008, Low-Noise Systems in the Deep Space Network, Jet Propulsion Laboratory California Institute of Technology.

[38] K. N. KOCHARYAN, 1979, The zero-field splitting of the $\mathrm{Mo}^{3+}$ ground state in corundum. Physica status solidi (b), 94(2) : K129K131.

[39] E. G. SHAROYAN, O. S. TOROSYAN, E. A. MARKOSYAN and V. T. GABRIELYAN, 1974, EPR and spin-lattice relaxation of $\mathrm{Mo}^{3+}$ ions in corundum. Physica status solidi (b), $65: 773778$. 